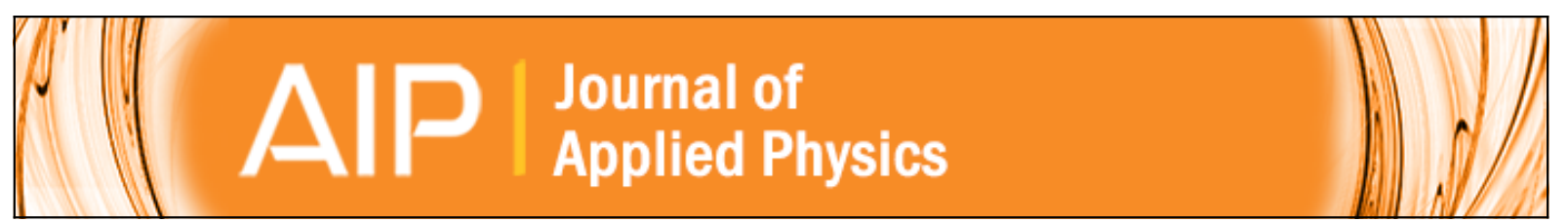

Experimental and theoretical study of the dynamics of self-sustained oscillations in a standing wave thermoacoustic engine

M. Guedra, G. Penelet, and P. Lotton

Citation: Journal of Applied Physics 115, 024504 (2014); doi: 10.1063/1.4861879

View online: http://dx.doi.org/10.1063/1.4861879

View Table of Contents: http://scitation.aip.org/content/aip/journal/jap/115/2?ver=pdfcov

Published by the AIP Publishing

$\underset{\substack{\text { Publoning } \\ \text { PIP }}}{A}$ Re-register for Table of Content Alerts

Create a profile.

Sign up today! 


\title{
Experimental and theoretical study of the dynamics of self-sustained oscillations in a standing wave thermoacoustic engine
}

\author{
M. Guedra, ${ }^{\text {a) }}$ G. Penelet, and P. Lotton \\ Laboratoire d' Acoustique de l'Université du Maine (UMR CNRS 6613), Avenue Olivier Messiaen, \\ 72085 Le Mans Cedex 9, France
}

(Received 17 September 2013; accepted 27 December 2013; published online 10 January 2014)

\begin{abstract}
A model for the description of the transient regime leading to steady-state sound in a quarterwavelength thermoacoustic prime mover is proposed, which is based on the description of the unsteady heat transfer in the system, coupled with an ordinary differential equation describing wave amplitude growth/attenuation. The equations are derived by considering a cross-sectional averaged temperature distribution along the resonator, and by assuming that both the characteristic time associated with heat diffusion through the stack and that associated with the thermoacoustic amplification are much larger than the acoustic period. Attention is here focused on the only mechanism of saturation due to heat transport by sound within the stack. The numerical solving of the governing equations leads to the prediction of the transient regime, which is compared with experimental results for several values of the heat power supplied to the system and for several positions of the stack in the resonator. The model reproduces the experiments quite well, notably showing that a small diminution of the temperature in the vicinity of the hot end of the stack is associated to an overshoot of wave amplitude growth, while heat diffusion through the whole stack impacts the subsequent evolution of wave amplitude leading to steady state. Additional experimental results exhibiting complicated regimes of wave amplitude evolution are provided, which are not reproduced by the present model. (C) 2014 AIP Publishing LLC.
\end{abstract}

[http://dx.doi.org/10.1063/1.4861879]

\section{INTRODUCTION}

Thermoacoustic prime movers convert heat into acoustical work. They generally consist of an acoustic resonator partially filled with an open-cells porous material, often referred to as the stack. When a sufficient temperature gradient is applied along this stack, in such a way that it goes beyond the onset threshold, a high-amplitude self-sustained acoustic wave is generated. One of the interesting features of this kind of engine is that the energy conversion is based on the oscillations of a fluid and therefore does not require any moving mechanical pieces (piston and crankshaft). For almost 30 years, many thermoacoustic systems have thus been studied in order to improve their efficiency, and the effect of most of the elements on the operation of thermoacoustic engines has been analyzed, such as the shape of the resonator, the characteristics of the porous material, the working fluid or the heat exchangers. ${ }^{1}$ Designing thermoacoustic systems generally lies on the linear theory of thermoacoustics, ${ }^{2}$ which provides reasonable agreement between the predicted performances and those actually achieved once steady-state is reached. ${ }^{3,4}$ However, these design tools based on the linear theory are not devoted to the description of the initial build-up of self-oscillations, but to the prediction of the operating point of the engine under an assigned (generally linear) temperature distribution along the thermoacoustic

\footnotetext{
${ }^{a)}$ Present address: Université Pierre et Marie Curie, Institut Jean Le Rond d'Alembert (UMR CNRS 7190), 4 place Jussieu, 75252 Paris cedex 05, France. Electronic mail: matthieu.guedra@dalembert.upmc.fr
}

core. Moreover, several works in the literature have reported experimental observations exhibiting complex dynamics occurring during the transient regime of wave amplitude growth, such as the periodic and spontaneous onset/damping of acoustic oscillations, ${ }^{5-8}$ the double-threshold effect, ${ }^{9}$ or the fishbone-like instability. ${ }^{10}$ The above mentioned effects are due to a competition between thermoacoustic amplification and various nonlinear processes (heat transport by sound, edge effects, nonlinear acoustic propagation, etc.), and it is therefore challenging to account for the latter effects in order to reproduce the dynamics observed in experiments.

Several works have been devoted to the description of the transient regime of wave amplitude growth and its saturation due to nonlinear processes in thermoacoustic systems. Some of them have focused attention on the description of the cascade process of higher harmonics generation in standing wave prime-movers submitted to an assigned temperature difference, the governing equations being solved either by direct numerical simulation $^{11-13}$ or analytically using a method of multiple time scales. ${ }^{14}$ Other studies have described the transient regime under an assigned heat input (instead of a temperature difference) in different kinds of engines (e.g., annular engine, ${ }^{7,15}$ standing-wave engine, ${ }^{8}$ or thermoacoustic-Stirling engine ${ }^{16}$ ): in these models, sound saturation occurs via the diminution of the temperature gradient due to heat transport by the thermoacoustic effect along the stack, while the propagation of acoustic waves is assumed linear, and it is described either by lumped elements ${ }^{16}$ or by two-ports. ${ }^{17}$ Attempts have also been made (in the above mentioned two-port models) to account for heat convection by acoustic streaming in an annular thermoacoustic 
engine, ${ }^{7,15}$ or more recently in the standing wave thermoacoustic engine, ${ }^{8}$ which will be studied in the following. It is also worth mentioning that a few papers dealt with the use of commercial computational fluid dynamics simulation tools to compute the transient regime in thermoacoustic devices of complicated geometry, ${ }^{18,19}$ leading to results that are still quite different from experiments. Although time consuming, these latter approaches might be, ultimately, appropriate to describe thermoacoustic engines accurately, but it is still of interest to pursue the investigation of the transient regime by means of a simplified modeling in order to get a deeper insight about the dominant mechanisms controlling the saturation of the acoustic wave. Finally, it is worth mentioning that only a few of the studies mentioned above provide a direct and exhaustive comparison with experiments, and it is therefore the main scope of this paper to provide such a comparison, in the simplest of the existing thermoacoustic oscillators.

The purpose of the present paper is to propose a modeling of the transient regime of the thermoacoustic instability in a quarter-wavelength thermoacoustic prime-mover working with air at atmospheric pressure. This device has already been studied recently in Ref. 8 , in which both experimental data and a very simplified modeling of the transient regime are provided. However, contrarily to the works of Ref. 8 in which the equations governing unsteady heat transfer are simplified into a set of ordinary differential equations, the influence of the amplitude and the shape of the instantaneous temperature distribution on the dynamics of wave amplitude growth are investigated here. The influence of acoustic streaming (as well as that of other nonlinear processes) is discarded in the frame of this study (notably because its accurate description is still arduous), and the main purpose of these works is therefore to evaluate the impact of the only acoustically enhanced thermal diffusivity of the stack through a quantitative comparison with experiments. After a brief description of the experimental apparatus, both the modeling and the procedure for the numerical simulation of the transient regime are presented in Sec. II. Experimental and theoretical results are compared in Sec. III, and further discussions are proposed concerning the experimental observations, which are not reproduced by the model.

\section{THEORY}

The thermoacoustic engine under study consists of a cylindrical glass tube partially filled with the stack. ${ }^{8,20}$ A schematic drawing of this device is presented in Fig. 1. The resonator has a length $L=0.49 \mathrm{~m}$ and an inner radius $R=2.6 \mathrm{~cm}$, and it is closed at one end with a rigid plug, inside which a microphone (labelled as $\mathrm{P}$ in Fig. 1) is flush mounted. The stack consists of a cylinder (radius $R$ and length $l_{s}=4.8 \mathrm{~cm}$ ), which is made up of a ceramic catalyst with many square channels of semi-width $r_{s}=0.45 \mathrm{~mm}$. Heat is supplied to the system by means of a nichrome wire (36 cm in length, $0.25 \mathrm{~mm}$, resistivity $7 \Omega / f t$ at $300 \mathrm{~K}$ ) connected to a DC electrical power supply. Temperature measurements are performed by using three type $\mathrm{K}$ thermocouple probes placed along the axis of the waveguide at the center of the stack $\left(T_{1}\right)$, at the hot interface $\left(T_{2}\right)$, and inside the

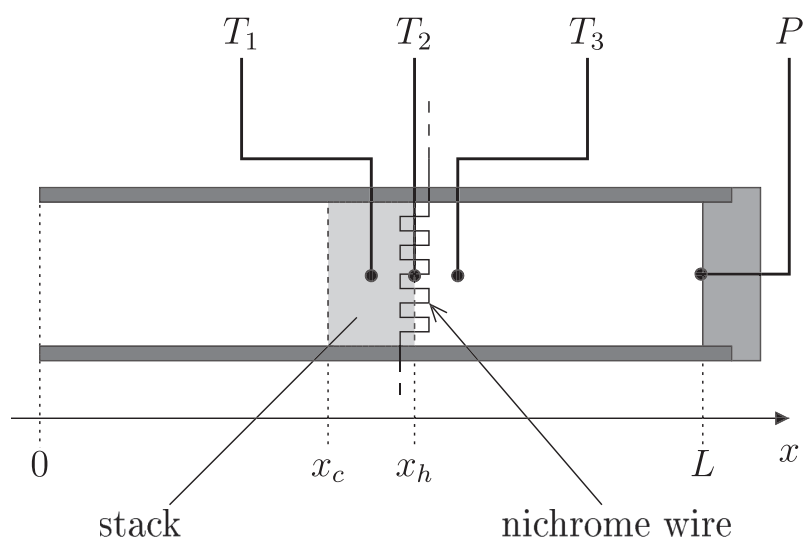

FIG. 1. Schematic drawing of the experimental apparatus.

resonator $\left(T_{3}\right)$ at a distance $d=l_{s} / 2$ from the hot interface of the stack, respectively (see Fig. 1).

Applying an amount of heat $Q$ to the stack via the nichrome wire results in heat diffusion through the system described in Fig. 1, which may lead to the amplification of a sound wave in the resonator. In order to describe the instantaneous amplification or attenuation of the acoustic pressure amplitude, the first step thus consists in describing heat transport through the system. Apart from the surrounding glass walls, the system consists of two different media, namely, the stack of porosity $\phi=0.73$ and the remaining of the resonator filled with air. The stack is considered as an equivalent fluid medium of thermophysical properties

$$
\begin{gathered}
\lambda_{s}=\phi \lambda_{a}+(1-\phi) \lambda_{c}, \\
\rho_{s} C_{s}=\phi \rho_{a} C_{a}+(1-\phi) \rho_{c} C_{c},
\end{gathered}
$$

where $\lambda, \rho$, and $C$ are the thermal conductivity, the density, and the isobaric specific heat of the medium, respectively, and the subscripts $s, a$, and $c$ are used for the stack, the air, and the ceramic frame, respectively.

Note that the walls of the resonator are assumed to rest at constant temperature $T_{\infty}=300 \mathrm{~K}$, as well as both ends of the device

$$
T(0, t)=T(L, t)=T_{\infty}, \quad \forall t \geq 0,
$$

and that the heat diffusion is supposed to occur along the axial direction $x$. The mean (i.e., non-oscillating) axial temperature $T$ along the whole device is then described by the following set of one-dimensional partial differential equations (related to each part of the engine):

$$
\begin{gathered}
\forall x \in\left[0, x_{c}\right] \cup\left[x_{h}, L\right], \\
\frac{\partial T}{\partial t}=\frac{1}{\rho_{a} C_{a}} \frac{\partial}{\partial x}\left(\lambda_{a} \frac{\partial T}{\partial x}\right)-\frac{T-T_{\infty}}{\tau_{a}}, \\
\forall x \in\left[x_{c}, x_{h}\right], \\
\frac{\partial T}{\partial t}=\frac{1}{\rho_{s} C_{s}} \frac{\partial}{\partial x}\left(\lambda_{s} \frac{\partial T}{\partial x}\right)-\frac{T-T_{\infty}}{\tau_{s}}-\frac{1}{\rho_{s} C_{s}} \frac{\partial \varphi_{a c}}{\partial x},
\end{gathered}
$$

where the term $\partial_{x} \varphi_{a c}$ describes the advective heat transport by sound resulting from the interaction of the oscillating gas 
with the stack walls, and where the two characteristic times $\tau_{a}$ and $\tau_{s}$ take into account the heat exchange with the surrounding walls. These characteristic times can be estimated from empirical correlations in steady flow, ${ }^{23}$ leading to the following analytical expressions (see also the appendix of Ref. 15 for more details):

$$
\begin{gathered}
\tau_{a}=\frac{R^{2}}{3.66} \frac{\rho_{a} C_{a}}{\lambda_{a}} \\
\tau_{s}=\frac{\ln (2)}{2} \rho_{s} C_{s} R^{2} \\
\times\left[\frac{1-\sqrt{\Phi}}{\lambda_{c}}+\left(14.64 \lambda_{a}+\lambda_{c} \frac{1-\sqrt{\Phi}}{\sqrt{\Phi}}\right)^{-1}\right] .
\end{gathered}
$$

If the spatial distribution of both the temperature field $T(x, t)$ and the acoustic field are known at a given time, the instantaneous thermoacoustic heat flux $\varphi_{a c}$ can be calculated. ${ }^{2,22}$ More precisely, it is defined as

$$
\varphi_{a c}=\frac{\rho_{a} T}{2} \Re\left(\left\langle\tilde{s} \tilde{v}_{x}^{*}\right\rangle\right),
$$

where $\Re(\ldots)$ denotes the real part of a complex number, * is the conjugate of a complex number, $\langle\ldots\rangle$ denotes crosssectional averaging, and $\tilde{s}$ and $\tilde{v}_{x}$ are the complex amplitudes of the acoustic entropy per unit mass and the axial acoustic velocity, respectively. Considering the propagation of harmonic plane waves, $\tilde{s}$ and $\tilde{v}_{x}$ can be expressed as functions of the acoustic pressure $\tilde{p}$ and its longitudinal gradient under the following forms:

$$
\begin{gathered}
\tilde{s}=-\frac{\tilde{p}}{\rho_{a} T} F_{\nu}-\frac{C_{a}}{\omega^{2} \rho_{a} T} \frac{\partial \tilde{p}}{\partial x} \frac{\partial T}{\partial x}\left[1-\frac{\operatorname{Pr} F_{\nu}-F_{\kappa}}{\operatorname{Pr}-1}\right], \\
\tilde{v}_{x}=\frac{1}{i \omega \rho_{a}} \frac{\partial \tilde{p}}{\partial x}\left[1-F_{\nu}\right],
\end{gathered}
$$

where $\operatorname{Pr}$ is the Prandtl number of the fluid and the wellknown functions $F_{\nu}$ and $F_{\kappa}$ characterize the viscous and the thermal coupling between the oscillating fluid and the stack's channel. Analytical expressions for $F_{\nu}$ and $F_{\kappa}$ are available for various geometries of channels (e.g., in Ref. 24).

The external heat source $Q(t)$ is assumed to be uniformly applied on the cross-sectional area at position $x=x_{h}$, and the continuity of heat fluxes at $x=x_{c}$ and $x=x_{h}$ leads to the following relations:

$$
\begin{gathered}
\left.\lambda_{a} \frac{\partial T}{\partial x}\right|_{x_{c}^{-}}=\left.\lambda_{s} \frac{\partial T}{\partial x}\right|_{x_{c}^{+}}-\varphi_{a c}\left(x_{c}^{+}\right), \\
\left.\lambda_{s} \frac{\partial T}{\partial x}\right|_{x_{h}^{-}}=\left.\lambda_{a} \frac{\partial T}{\partial x}\right|_{x_{h}^{+}}+\varphi_{a c}\left(x_{h}^{-}\right)+\frac{Q(t)}{\pi R^{2}} .
\end{gathered}
$$

The set of differential equations (4) and (5) combined with the boundary conditions (3), (11), and (12) describe the variations of the temperature distribution $T(x, t)$, which also depend on the acoustic field through the thermoacoustic heat flux $\varphi_{a c}$.
The second step in the derivation of the model consists in calculating the acoustic field which itself is controlled by the instantaneous temperature distribution. To that purpose, we use a method described in previous papers, ${ }^{8,17}$ which is based on the transfer matrices formalism and on the use of a complex frequency whose imaginary part describes wave amplification. This method is briefly described in the following with reference to previous papers for more details. The relationship between the complex amplitudes of acoustic pressure $\tilde{p}(x)$ and mean velocity $\left\langle\tilde{v}_{x}(x)\right\rangle$ at both ends of the engine can be written as follows:

$$
\begin{aligned}
\left(\begin{array}{c}
\tilde{p}(L) \\
\left\langle\tilde{v}_{x}(L)\right\rangle
\end{array}\right) & =\mathbf{A}_{\mathbf{2}} \times \mathbf{A}_{\mathbf{s}} \times \mathbf{A}_{\mathbf{1}} \times\left(\begin{array}{c}
\tilde{p}(0) \\
\left\langle\tilde{v}_{x}(0)\right\rangle
\end{array}\right), \\
& =\left(\begin{array}{cc}
a_{11} & a_{12} \\
a_{21} & a_{22}
\end{array}\right) \times\left(\begin{array}{c}
\tilde{p}(0) \\
\left\langle\tilde{v}_{x}(0)\right\rangle
\end{array}\right),
\end{aligned}
$$

where $\mathbf{A}_{\mathbf{1}}, \mathbf{A}_{\boldsymbol{s}}$, and $\mathbf{A}_{\mathbf{2}}$ are the transfer matrices describing the acoustic propagation in each region defined as $x \in\left[0, x_{c}\right]$, $x \in\left[x_{c}, x_{h}\right],{ }^{26}$ and $x \in\left[x_{h}, L\right]$, respectively (see Eq. (19) in Ref. 25). Neglecting acoustic radiation at the open end of the engine $(\tilde{p}(0)=0)$ and considering that the plug at the other end is rigid $\left(\left\langle\tilde{v}_{x}(L)\right\rangle=0\right)$, the following characteristic equation of the thermoacoustic system is obtained:

$$
a_{22}=0 \text {. }
$$

If the temperature distribution $T(x)$ is known at time $t$, it can be used as an input parameter to calculate $a_{22}$ as a function of the angular frequency $\omega$, but it is generally not possible to find a real angular frequency $\omega$ so that $a_{22}=0$. This problem is fixed here by assuming in Eq. (15) that $\omega=\Omega+i \alpha$ is a complex angular frequency ${ }^{17}$ whose real part $\Omega$ represents the angular frequency of acoustic oscillations, while the imaginary part $\alpha$ describes wave amplitude growth. The validity of such an approach is actually based on the existence of two different time scales: a fast time scale corresponding to the period of acoustic oscillations, and a slow one corresponding to the evolution of the amplitude of the wave. The present theoretical modeling relies on the separation of these two time scales and therefore on the quasi-steady state assumption stating that the acoustic pressure amplitude and the mean (i.e., non-oscillating) temperature in the device can be considered as constant during a few acoustic periods ${ }^{17}$ $(\alpha \ll \Omega)$. From this assumption, it is therefore quite direct to describe the slow variations of the acoustic pressure amplitude at any point $x$ and especially at position $x=L$ with a simple ordinary differential equation as follows:

$$
\frac{d P}{d t}-\alpha P=0
$$

where $P=|\tilde{p}(L)|$ and the amplification rate $\alpha$ is calculated at each time-step by solving the characteristic equation (15), using as an input parameter the known instantaneous temperature distribution $T(x, t)$. Moreover, the knowledge of the instantaneous peak amplitude $P$ allows to calculate the spatial distribution of other acoustic variables, as well as the thermoacoustic heat flux $\varphi_{a c}$. 
Experiments were performed on the present device: the procedure consists in starting the measurements for the whole system at room temperature $T_{\infty}$, a heat input power $Q$ being supplied to the system at time $t=0$ (note that this procedure is slightly different from the one used in Ref. 8 , in which an initial heat supply $Q_{0}$ is provided to the system so that it is just below threshold, and an increment $\Delta Q$ is then supplied to initiate acoustic oscillations). The same procedure is used in the numerical solving, which is proceeded within two steps as explained below.

(1) At time $t=0$, the acoustic pressure amplitude is fixed at $P=0.5 \mathrm{~Pa}$ (quantification step of the acquisition card) and is kept constant until (step (2)). The amount of heat power $Q$ is applied and the heat transfer model defined by Eqs. (3)-(5) and Eqs. (11) and (12) is solved using a Crank-Nicholson numerical scheme. ${ }^{27}$ In order to have sufficient accuracy, the ratio between the spatial step and the total length of the stack is set to $1 / 80$. The solving of the characteristic equation (15) is realized at each time step by taking the instantaneous temperature distribution $T(x, t)$ as an input parameter in order to calculate the complex frequency $\omega$. As the term $a_{22}$ on the left hand side of Eq. (15) comes from the product of three transfer matrices involving infinite series of integral operators, ${ }^{15}$ the angular frequency of acoustic oscillation $\Omega=\Re(\omega)$ and the amplification rate $\alpha=\Im(\omega)$ are obtained by equating $a_{22}$ to 0 using a Newton-Raphson method. ${ }^{17}$

(2) When the amplification rate $\alpha$ is just above 0 (above the onset of the thermoacoustic instability), the numerical integration of the differential equation for acoustic pressure amplitude (16) is realized with a 4th-order RungeKutta method, combined with an optimization procedure controlling the time step. The numerical computation of $T(x, t)$ and $\alpha$ is then realized as in step (1) except that the heat transport by sound, $\varphi_{a c}$, is taken into account.

\section{COMPARISON BETWEEN THEORY AND EXPERIMENTS}

In the following, we present several comparisons between experimental and theoretical results for the evolutions of both the acoustic pressure amplitude $P$ and the temperatures $T_{i}(i=1,2,3)$ during the transient regime of wave amplitude growth. This implies to define the numerical values of the thermophysical parameters used for the computation, but it is worth mentioning that some of these parameters are actually known with poor accuracy. This notably concerns the thermophysical parameters of the stack's material (cordierite), for which we only found a few data (see the second column of Table. I), provided without warranty (e.g., in http://www.ferroceramic.com). This is also the case for the characteristic times $\tau_{a}$ and $\tau_{s}$, which are only roughly estimated [see Eqs. (6)] from empirical correlations associated to forced convection within a duct in the limit of a vanishing steady flow velocity. Due to this, we considered that it was acceptable to adjust our estimated values of these parameters in order that theoretical results be slightly closer
TABLE I Values of the thermophysical parameters (given at $300 \mathrm{~K}$ ) used for the calculations. The second column corresponds to the estimates of each parameter, while the third column to those used for computations. The estimated values of the stack's material (cordierite) are those provided by FerroCeramic Grinding, Inc. (see http://www.ferroceramic.com). The parameters $\rho_{a} C_{a}, \lambda_{a}, \tau_{a}$, and $\tau_{s}$ depend on the temperature via the dependence of both $\rho_{a}$ (ideal gas law) and $\lambda_{a}\left(\lambda_{a} \propto T^{\beta}\right.$, with $\beta=0.77$ (Ref. 21)).

\begin{tabular}{lcc}
\hline \hline Parameter & Estimated value & Retained value \\
\hline$\rho_{a} C_{a}\left(\mathrm{~J} \mathrm{~m}^{-3} \mathrm{~K}^{-1}\right)$ & $1.2 \times 10^{3}$ & Unchanged \\
$\rho_{c} C_{c}\left(\mathrm{~J} \mathrm{~m}^{-3} \mathrm{~K}^{-1}\right)$ & $3.8 \times 10^{6}$ & Divided by $4 / 3$ \\
$\lambda_{a}\left(\mathrm{~W} \mathrm{~m}^{-1} \mathrm{~K}^{-1}\right)$ & $2.26 \times 10^{-2}$ & Unchanged \\
$\lambda_{c}\left(\mathrm{~W} \mathrm{~m} \mathrm{~K}^{-1}\right)$ & 3 & Unchanged \\
$\tau_{a}(\mathrm{~s})$ & Eq. (6) & $\frac{1}{2} \times$ Eq. (6) \\
$\tau_{s}(\mathrm{~s})$ & Eq. (7) & $2 \times$ Eq. (7) \\
\hline \hline
\end{tabular}

to experiments. Therefore, and accordingly with the data of Table I, we chose to multiply the initial estimates of $\tau_{s}$ and $\tau_{a}$ by factors 2 and $1 / 2$, respectively, and to divide the specific heat $\rho_{c} C_{c}$ by $4 / 3$, leading to the final set of input parameters defined in the third column of Table I. This adjustment has been carried on by trial and error, in such a way that the calculated time of occurrence of the exponential growth of the thermoacoustic instability after switching on the heat supply coincides with the one observed in experiments. Note that another approach for estimating the parameters of Table I more accurately would have been to measure the transfer matrix $\mathbf{A}_{\mathbf{2}} \times \mathbf{A}_{\boldsymbol{s}} \times \mathbf{A}_{\mathbf{1}}$ of the thermoacoustic prime-mover ${ }^{28,29}$ as a function of the frequency and the heat input $Q$, and to proceed to data fitting from the comparison of the theoretical transfer matrix with the one obtained from experiments.

The results obtained for a stack at position $x_{h}=0.29 \mathrm{~m}$ and for a heat supply fixed to $Q=33.3 \mathrm{~W}$ are presented in Fig. 2. The instantaneous acoustic pressure measured by the microphone is shown with grey shading [Fig. 2(a)]: the initial and very quick exponential growth of the wave leads to an overshoot around $t \approx 40 \mathrm{~s}$, which is followed by an increase of acoustic pressure up to $t \approx 100 \mathrm{~s}$, and a slow decay before reaching steady-state. The corresponding temperature measurements are presented with dashed lines in Figs. 2(b)-2(d): one can notably observe that the amplification of the sound wave impacts the temperature variations at the hot stack end (temperature $T_{2}$ ), due to the acoustically induced enhancement of heat transfer along the porous sample. The calculated evolutions of both pressure $P$ and temperatures $T_{i}$ are presented with solid lines in Fig. 2. From the analysis of Fig. 2(a), it appears that the model succeeds in reproducing the evolution of the acoustic pressure amplitude (and notably the occurrence of the overshoot), although the calculated steady-state amplitude of self-sustained oscillations is under-estimated by the model. Note that if we had used the "initial" values for $\rho_{c} C_{c}, \tau_{s}$, and $\tau_{a}$ (second column of Table I), then the results obtained would not differ significantly from those of Fig. 2, but the time required for the onset of thermoacoustic instability would have been over-estimated (i.e., $t \approx 52 \mathrm{~s}$ instead of $40 \mathrm{~s}$ ), and the steady-state magnitude of acoustic pressure would be less than 5\% higher than that of Fig. 2. From the comparative 

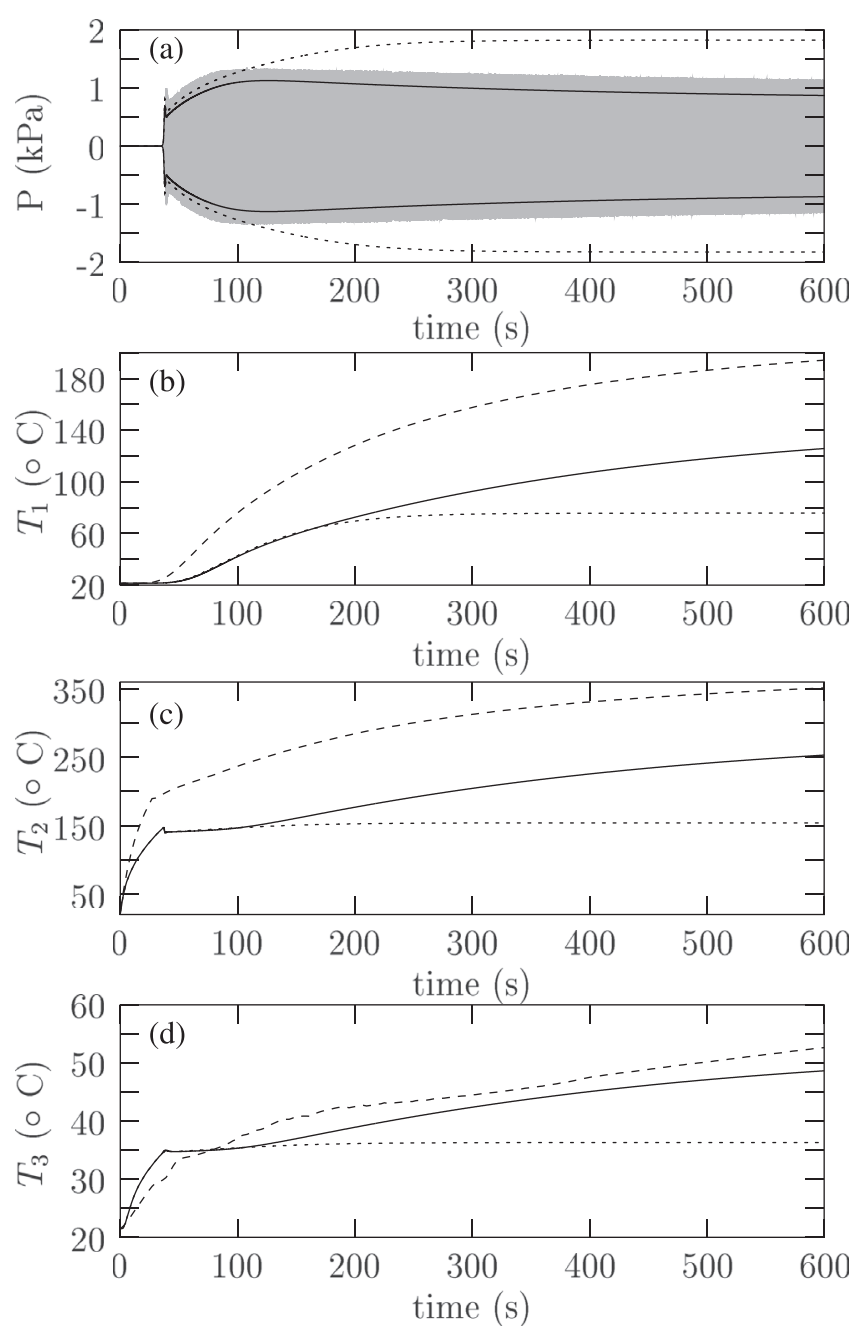

FIG. 2. Transient regime of wave amplitude growth obtained for $x_{h}=0.29 \mathrm{~m}$ and $Q=33.3 \mathrm{~W}$. Both the evolution of acoustic pressure (a) and of the temperatures $T_{1-3}$ (b)-(d) are presented as a function of time. Experimental results are presented with grey shading for acoustic pressure, and with dashed lines for the temperatures $T_{i}$. Theoretical results are presented with solid lines and dotted lines. The results presented with solid lines are those for which the continuity of heat flux is considered at position $x=x_{c}$, while the results presented with dotted lines are those for which the temperature at $x=x_{c}$ is assigned to the room temperature $T_{\infty}$.

analysis of the temperature evolutions [Figs. 2(b)-2(d)], a qualitative agreement is also obtained, but the calculated $T_{i}$ are significantly lower than the measured ones. One of the possible reasons for these differences is that the description of unsteady heat transfer is based on a one-dimensional, simplified description of the phenomena. This notably means that the calculated $T_{i}$ correspond to cross-sectional averaged temperatures, while the measured $T_{i}$ are those along the median axis of the duct. Therefore, it is not surprising that the calculated temperatures in the stack are significantly lower than the measured ones since the former are cross-sectional averaged temperatures while the latter are temperatures along the axis of the waveguide (note that we measured the temperature close to the glass walls at position $x_{h}$, and found that it is several tens of Kelvin lower than that on the axis).

Additional simulation results are also presented with dotted lines in Fig. 2, which aim at pointing out the impact of the details of the variations of the temperature distribution on the thermoacoustic amplification process. These additional results are obtained for the same input parameters, but it is however assumed that the cold end of the stack is kept at room temperature $T_{\infty}$, Eq. (11) being thus replaced with the simpler condition that $T\left(x_{c}, t\right)=T_{\infty}$. Therefore, the heat diffusion (4) in the domain $x \in\left[0, x_{c}\right]$ is discarded and the matrix $\mathbf{A}_{\mathbf{1}}$ in Eq. (13) is the classical one of a simple duct. Although one would not expect, a priori, a significant difference between this calculation and the one presented with solid lines, the results obtained show that discarding the slow warming of the cold end of the stack strongly impacts the last part of the transient regime of wave amplitude growth. Clearly, the initial modeling of unsteady heat transfer at the cold stack end, i.e., Eq. (11), leads to a better reproduction of the dynamics observed in experiments.

In Fig. 3, the influence of the heating power and of the stack location on the onset and the saturation of the thermoacoustic instability are investigated. Figure 3(a) presents the dynamics of wave amplitude growth for two values of heat power $Q$, the stack being still positioned at $x_{h}=0.29 \mathrm{~m}$. The experimental results are shown with grey $(Q=18.6 \mathrm{~W})$ and soft-grey $(Q=23.3 \mathrm{~W})$ shadings, respectively. The corresponding theoretical results are shown with dashed-dotted $(Q=18.6 \mathrm{~W})$ and solid $(Q=23.3 \mathrm{~W})$ lines, respectively. From the analysis of Fig. 3(a), it appears that an increase of the heat power leads to a smaller onset time and a larger pressure level, as one would expect. The experimental results are well-reproduced by the model, with a good prediction of the occurrence of both the onset and the overshoot, as well as the subsequent evolution of acoustic pressure amplitude. The corresponding evolutions of the temperatures $T_{i}$ are not presented here, since the results obtained do not provide highly relevant additional information to the results presented in Fig. 2. The influence of the stack location on the thermoacoustic amplification process is presented in Fig. 3(b). The experimental results are shown with grey
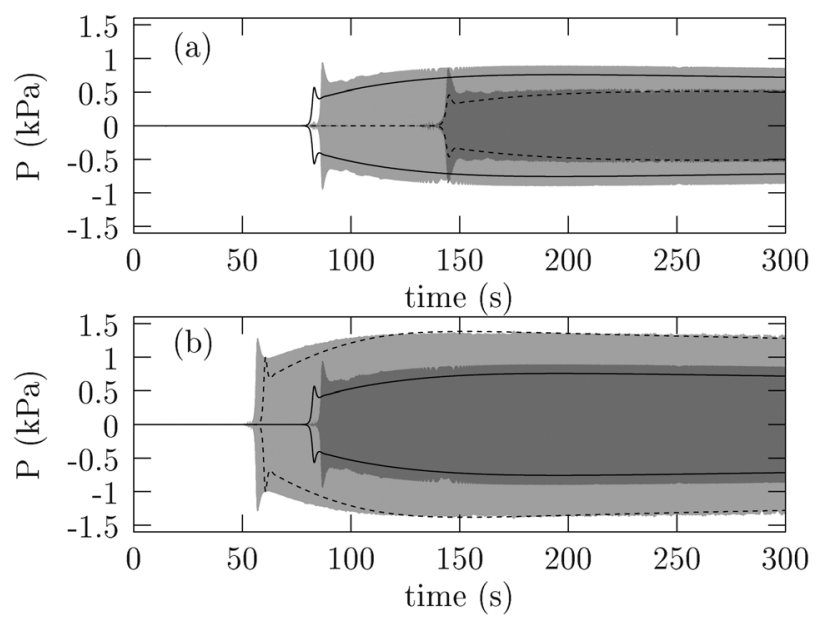

FIG. 3. (a) Effect of the heating power: experimental (shaded) and theoretical (lines) evolutions of acoustic pressure amplitude as a function of time, for $x_{h}=0.29 \mathrm{~m}$, and for $Q=18.6 \mathrm{~W}$ (grey shading and dashed-dotted lines) or $Q=23.3 \mathrm{~W}$ (soft grey shading and solid lines), respectively. (b) Effect of the stack position: experimental and theoretical evolutions of acoustic pressure amplitude as a function of time, for $Q=23.3 \mathrm{~W}$, and for $x_{h}=0.29 \mathrm{~m}$ (grey shading and solid lines) or $x_{h}=0.35 \mathrm{~m}$ (soft grey shading and dasheddotted lines), respectively. 

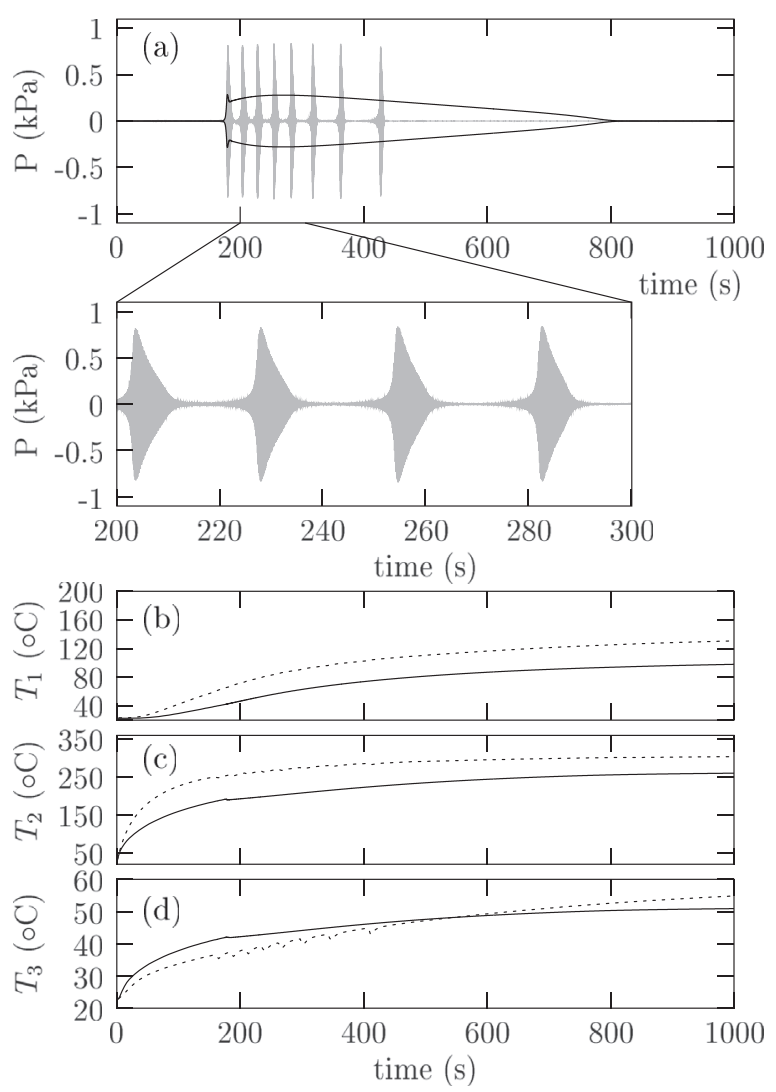

FIG. 4. Transient regime of wave amplitude growth obtained for $x_{h}=0.23 \mathrm{~m}$ and $Q=23.3 \mathrm{~W}$. Both the evolution of acoustic pressure (a) and of the temperatures $T_{1-3}$ (b)-(d) are presented as a function of time. Experimental results are presented with grey shading for acoustic pressure, and with dashed lines for the temperatures $T_{i}$. Theoretical results are presented with solid lines.

$\left(x_{h}=0.29 \mathrm{~m}\right)$ and soft grey $\left(x_{h}=0.35 \mathrm{~m}\right)$ shadings, respectively. The corresponding theoretical results are shown with solid and dashed-dotted lines, respectively. From the analysis of Fig. 3(b), it appears that the closer is the stack to the rigid end of the device, the higher is the acoustic pressure amplitude. Once again, experimental and theoretical results are in good agreement.

The results presented in Figs. 2 and 3 exhibit a transition to steady-state sound. There exist, however, particular conditions leading to more complicated processes, ${ }^{8,20}$ which are observed when the stack is placed closer to the open end of the resonator, and when the heat power is fixed around its critical value corresponding to the onset threshold. This phenomenon is investigated in the following. As an example, two sets of measurements of acoustic pressure and temperature variations are presented in Figs. 4 and 5, as well as the associated predictions derived from the theoretical model.

In Fig. 4, the transient regime is obtained for a stack position $x_{h}=0.23 \mathrm{~m}$ and for a heat power input $Q=23.3 \mathrm{~W}$. In both cases of experiment and theory, the supply of heat leads to the onset of self-sustained oscillations, which are, however, completely switched off after a few minutes. This means that the assigned value of heat input $Q=23.3 \mathrm{~W}$ is actually below its critical value corresponding to the marginal stability condition, but that self-sustained waves can be temporarily generated due to a favorable, but ephemerous, distribution of the temperature field during the process of heat diffusion through the stack. It is also clear from the analysis of Fig. 4 that the model does not reproduce the complicated dynamics observed in experiments, since the latter exhibit a succession of seven rapid onset and damping of acoustic oscillations before the final extinction, while only one long burst is observed in the simulations. Interestingly, experimental results show that the onset/damping is accompanied with small oscillations of the temperatures $T_{2}$ and $T_{3}$ [although those of $T_{2}$ are hardly visible in Fig. 4(c)], which are not reproduced by the model. Therefore, the observed bursts of onset/damping of thermoacoustic instability are not only due to a too low $Q$, but also to complicated interactions between the temperature and the acoustic fields in the vicinity of the hot stack end, which are not described by the model.

In Fig. 5, the results are obtained for the same position of the stack but for a slightly larger heat supply $(Q=26.2 \mathrm{~W})$. The simulation predicts steady-state sound after a long transient regime, while the experimental results show a much more complicated behavior: the initial exponential growth (and the overshoot) is followed by a gradual increase of acoustic pressure amplitude (until $t \approx 200 \mathrm{~s}$ ), then by a slow decrease until $t \approx 400 \mathrm{~s}$, and finally by a quite abrupt loss of stability, which gives rise to a spontaneous and periodic onset/damping of acoustic waves. However, contrarily to the experimental results of Fig. 4, the latter switch on/off process never stops, even after half an hour (and larger time periods), while the model predicts the stabilization of the acoustic pressure amplitude to $P \approx 60 \mathrm{~Pa}$.

The results presented in Figs. 4 and 5 clearly show the limits of the simple model presented in Sec. II, and appeal for a discussion about the mechanisms, which might be responsible for the complicated dynamics observed in experiments. In our opinion, it seems reasonable to admit that the phenomena could hardly be explained by some nonlinear processes that do not involve the interaction with the temperature field, like minor losses or nonlinear propagation. Therefore, if the latter effects are discarded, it is instructive to remind the main approximations of our modeling to get an idea of what should be investigated in future works. First, the calculated temperature field is one-dimensional, while it is clear in the present device that there exist large temperature gradients in the radial direction. This may impact our estimate of the thermoacoustic amplification rate $\alpha$, and it is also conceivable that the onset of thermoacoustic instability impacts the temperature field along the radial direction, as it does along $x$. Second, apart from the thermoacoustic heat transport by sound, which is taken into account here, the mechanisms by which acoustic oscillations impact heat and mass transport in the thermoacoustic core are poorly understood. In previous works, ${ }^{8}$ we proposed a very simplified modeling of heat convection by acoustic streaming, and concluded that this mechanism might be considered, as well as the complicated heat and mass transport phenomena associated to aerodynamical edge effects at both ends of the stack. Therefore, regarding the complexity of the processes mentioned above, it is 

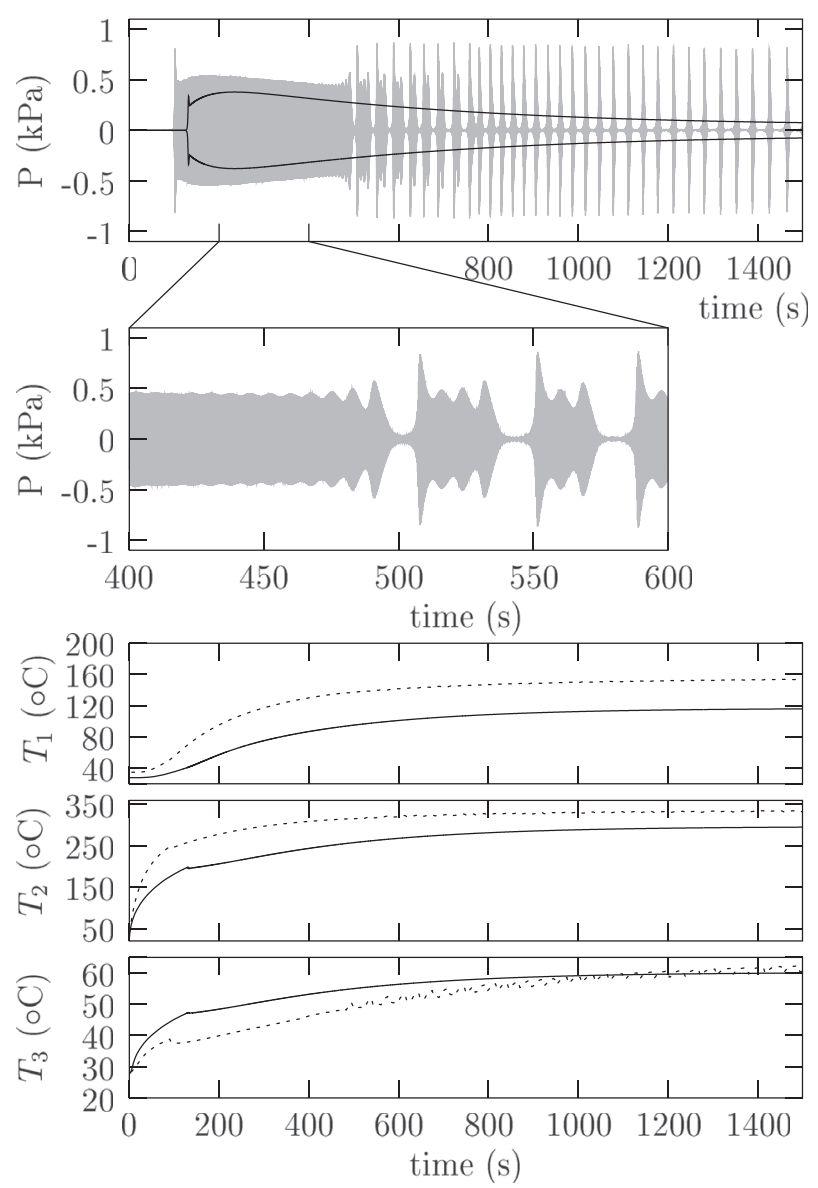

FIG. 5. Transient regime of wave amplitude growth obtained for $x_{h}=0.23 \mathrm{~m}$ and $Q=26.2 \mathrm{~W}$. Both the evolution of acoustic pressure (a) and of the temperatures $T_{1-3}$ (b)-(d) are presented as a function of time. Experimental results are presented with grey shading for acoustic pressure, and with dashed lines for the temperatures $T_{i}$. Theoretical results are presented with solid lines.

challenging to make further investigations in order to reproduce the experimental results of Figs. 4 and 5.

\section{CONCLUSION}

In this paper, we have presented a simplified, onedimensional modeling of the transient regime, leading to steady-state sound in a quarter-wavelength thermoacoustic prime-mover. Only the advective heat transport by sound due to the thermoacoustic process within the stack is taken into account to describe sound saturation, but the instantaneous variations of the details of the axial temperature distribution (and their impact on thermoacoustic amplification) are considered in this model. As a result, a good agreement between experiments and theory is obtained, at least under some circumstances. However, it is also shown that the model cannot reproduce the spontaneous and periodic onset/damping of the thermoacoustic instability, which is observed when the stack is placed closer to the open end of the resonator. This latter effect might be attributed to more complex interactions between the sound and the temperature fields. Considering that even the simplest of the thermoacoustic engines exhibit such complicated dynamics, it should be interesting, for more practical applications of thermoacoustics, to pursue the investigations about the mechanisms controlling the stability of sound waves above threshold.

${ }^{1}$ G. W. Swift, Thermoacoustics: A Unifying Perspective for Some Engines and Refrigerators (Acoustical Society of America, Melville NY, 2001).

${ }^{2}$ N. Rott, Adv. Appl. Mech. 20, 135 (1980).

${ }^{3}$ W. C. Ward and G. W. Swift, J. Acoust. Soc. Am. 123, 3546 (2008).

${ }^{4}$ W. C. Ward, G. W. Swift, and J. P. Clark, J. Acoust. Soc. Am. 95, 3671 (1994).

${ }^{5}$ G. W. Swift, J. Acoust. Soc. Am. 92, 1551 (1992).

${ }^{6}$ Z. B. Yu, Q. Li, X. Chen, F. Z. Guo, X. J. Xie, and J. H. Wu, Cryogenics 43, 687 (2003).

${ }^{7}$ G. Penelet, V. Gusev, P. Lotton, and M. Bruneau, Phys. Lett. A 351, 268 (2006).

${ }^{8}$ G. Penelet, M. Guedra, V. Gusev, and T. Devaux, Int. J. Heat Mass Transfer 55, 6042 (2012).

${ }^{9}$ G. Penelet, E. Gaviot, V. Gusev, P. Lotton, and M. Bruneau, Cryogenics 42, 527 (2002).

${ }^{10}$ Z. Yu, A. J. Jaworski, and A. S. Abduljalil, J. Acoust. Soc. Am. 128, EL188 (2010).

${ }^{11}$ H. Yuan, S. Karpov, and A. Prosperetti, J. Acoust. Soc. Am. 102, 3497 (1997).

${ }^{12}$ M. F. Hamilton, Y. A. Ilinskii, and E. A. Zabolotskaya, J. Acoust. Soc. Am. 111, 2076 (2002)

${ }^{13}$ S. Karpov and A. Prosperetti, J. Acoust. Soc. Am. 112, 1431 (2002).

${ }^{14}$ S. Karpov and A. Prosperetti, J. Acoust. Soc. Am. 107, 3130 (2000).

${ }^{15}$ G. Penelet, V. Gusev, P. Lotton, and M. Bruneau, Phys. Rev. E 72, 016625 (2005).

${ }^{16}$ A. T. A. M. de Waele, J. Sound Vib. 325, 974 (2009).

${ }^{17}$ M. Guedra and G. Penelet, Acta Acust. Acust. 98, 232 (2012).

${ }^{18}$ J. A. L. A. Nijeholt, M. E. H. Tijani, and S. Spoelstra, J. Acoust. Soc. Am. 118, 2265 (2005).

${ }^{19}$ G. Y. Yu, E. C. Luo, W. Dai, and J. Y. Hu, J. Appl. Phys. 102, 074901 (2007).

${ }^{20}$ G. Penelet and T. Biwa, Am. J. Phys. 81, 290 (2013).

${ }^{21}$ H. Bailliet, V. Gusev, R. Raspet, and R. Hiller, J. Acoust. Soc. Am. 110, 1808 (2001).

${ }^{22}$ T. Yazaki, A. Tominaga, and Y. Narahara, J. Heat Transfer 105, 889 (1983).

${ }^{23}$ F. P. Incropera and D. P. DeWitt, Introduction to Heat Transfer (John Wiley \& Sons, New York, 1985).

${ }^{24}$ W. P. Arnott, H. E. Bass, and R. Raspet, J. Acoust. Soc. Am. 90, 3228 (1991).

${ }^{25}$ G. Penelet, S. Job, V. Gusev, P. Lotton, and M. Bruneau, Acta Acust. Acust. 91, 567 (2005).

${ }^{26}$ V. Gusev, H. Bailliet, P. Lotton, and M. Bruneau, Acta Acust. Acust. 86, 25 (2000).

${ }^{27}$ M. N. Ozisik, Finite Difference Methods in Heat Transfer (CRC Press, 1994).

${ }^{28}$ M. Guedra, G. Penelet, P. Lotton, and J. P. Dalmont, J. Acoust. Soc. Am. 130, 145 (2011).

${ }^{29}$ F. C. Bannwart, G. Penelet, P. Lotton, and J. P. Dalmont, J. Acoust. Soc. Am. 133, 2650 (2013). 